\title{
El efecto de la conservación de recursos sobre la intención emprendedora en el contexto de crisis económica: el rol moderador de la autoeficacia y la creatividad
}

\author{
Pedro F. Salazar-Carvajal ${ }^{1}$, Isabel M. Herrera-Sánchez ${ }^{2}$, Samuel Rueda-Méndez ${ }^{2}$ y José M. León-Rubio ${ }^{2}$ \\ ${ }^{1}$ Secretaría de Desarrollo Social y Político. Gobernación del Quindio, Colombia \\ ${ }^{2}$ Departamento de Psicología Social. Universidad de Sevilla, España
}

\begin{abstract}
Resumen: Este estudio plantea una línea de investigación del emprendimiento que incorpora el análisis de factores personales y del contexto socioeconómico actual en el marco de la Teoría de la Conservación de Recursos. Los objetivos del estudio fueron analizar el efecto que ejercen la pérdida, amenaza de pérdida y ganancia de recursos sobre la intención emprendedora, y determinar el papel moderador de la autoeficacia emprendedora y la percepción de la propia creatividad. Se realizó un estudio transversal en 278 estudiantes universitarios de posgrado. Se comprobó que la pérdida y la ganancia de recursos contribuyeron en la decisión de crear una empresa y que el efecto positivo de ambas variables fue mayor cuando se unía el efecto de la percepción creativa, siendo la autoeficacia emprendedora la variable que presentó mayor fuerza predictiva. Se concluye que la Teoría de la Conservación de Recursos constituye un soporte teórico que permite comprender el emprendimiento en el contexto de crisis económi$\mathrm{ca}$, si bien se precisa la realización de estudios que permitan clarificar y consolidar los resultados obtenidos, incorporando análisis prospectivos que contemplen, la estructura social, las estrategias de afrontamiento, la perspectiva de género y los tipos de conducta de emprendimiento.

Palabras clave: estrés; autoeficacia emprendedora; creatividad; intención emprendedora; crisis económica.
\end{abstract}

\section{Introducción}

Este estudio explora factores que pueden estar relacionados con la decisión de crear una empresa en un contexto adverso como la crisis económica actual. En este análisis consideramos clave la Teoría de la Conservación de Recursos de Hobfoll $(1998$; 2001) por su funcionalidad y pertinencia al situar los eventos asociados a las pérdidas o ganancias de recursos en la experiencia de estrés. Se plantean como objetivos del estudio analizar la intención emprendedora en estudiantes universitarios de posgrado considerando la influencia que pueden ejercer la pérdida real o potencial de los recursos y su ganancia en el contexto de crisis económica, y determinar el papel moderador de la autoeficacia emprendedora y la percepción de la propia creatividad.

\section{Emprendimiento e incertidumbre}

El emprendimiento es un proceso que implica la identificación y el aprovechamiento de oportunidades para llevar adelante un proyecto empresarial en un contexto de cierto grado de incertidumbre (Mitchell et al., 2007), al mismo tiempo que constituye un factor preponderante para el desarrollo económico y la prosperidad de una sociedad (Landström, 2008). Por esta razón, se entiende que los investigado-

* Dirección para correspondencia [Correspondence address]: Isabel María Herrera Sánchez. Facultad de Psicología. Universidad de Sevilla. C/ Camilo José Cela s/n. 41018, Sevilla (España). E-mail: iherrera@us.es
Title: The effect of conservation of resources on the entrepreneurial intention in the context of economic crisis: the moderating role of the selfefficacy and creativity.

Abstract: This study presents a research line of entrepreneurship incorporating personal factors and current socio-economic context in the framework of the Conservation of Resources Theory. The objectives were to analyze the effect of the loss, threat loss, and gain of the resources on entrepreneurial intention and to determine the moderating role of entrepreneurial self-efficacy and perception of own creativity. A cross-sectional study in 278 university postgraduate students was conducted. It was found that loss and gain of resources contributed in the decision to start a business, the positive effect of both variables was greater when the effect of the creative perception joined. Entrepreneurial self-efficacy showed more predictive power. It is concluded that the Conservation of Resources Theory is a theoretical support for the study of entrepreneurship in the context of economic crisis, however studies are needed to clarify and consolidate the results obtained, incorporating prospective analysis covering social structure, coping strategies, gender perspective and types of entrepreneurial behaviour.

Key words: stress; entrepreneurial self-efficacy; creativity; entrepreneurial intention; economic crisis.

res concentren su atención en saber por qué las personas eligen entre todas las alternativas posibles una carrera empresarial y cuáles son los factores que pueden estar relacionados, tópico que ha generado un extenso debate sobre cómo ha de enfocarse la investigación.

Las primeras investigaciones se orientaron a la identificación de las características que distinguen a la persona emprendedora de otra no emprendedora y que pueden ser relevantes para la actividad empresarial. Entre tales atributos se encuentran la necesidad de logro, el locus de control, la toma de riesgos, la tolerancia a la ambigüedad, la creatividad y la autonomía (Cromie, 2000). Estudios meta-analíticos han señalado cómo las variables de personalidad pueden jugar un rol importante en los procesos implicados en la emergencia de una empresa y su éxito posterior (Zhao, Seibert y Lumpkin, 2010). Sin embargo, se considera que la observación de los rasgos no es suficiente para explicar las decisiones que afectan a la elección de la carrera empresarial (Frank, Lueger y Korunka, 2007). Gartner (1988), desde un enfoque conductual, fue muy concluyente al señalar que la investigación orientada a la creación de una empresa ha de centrarse más en lo que realiza la persona emprendedora y menos en quién es. Los autores que han prestado más atención a la cognición social, han subrayado la necesidad de considerar la dinámica entre las personas y su entorno, al entender que el emprendimiento es una actividad que se produce en el contexto social a la vez que se encuentra en la representación cognitiva que las personas hacen con relación a la actividad emprendedora (Mitchell et al., 2002; Shaver y Scott, 1991). 
Con estas bases, tomó fuerza el estudio de los factores cognitivos involucrados en los procesos de decisión de la actividad empresarial. Estos factores afectan a las valoraciones, los juicios y las decisiones que son claves para la identificación de oportunidades, la creación de empresas y el crecimiento en general (Mitchell et al., 2002). Este enfoque plantea que la decisión de convertirse en empresario es el resultado de elecciones deliberadas hechas por las personas (Shaver y Scott, 1991). En esta acción voluntaria, los autores sitúan la intención de crear una empresa como un precursor indispensable de los comportamientos relacionados con el inicio de un proyecto empresarial (Baron, 2004; Krueger, 2000; Krueger, Reilly y Carsrud, 2000; Liñán y Chen, 2009; Thompson, 2009). La intención emprendedora se define como "la convicción auto-reconocida de una persona de que ella se propone crear un nuevo negocio y de manera consciente planea hacerlo en algún momento en el futuro" (Thompson, 2009, p. 676). En consecuencia, la intención emprendedora lleva implícita una decisión consciente y planificada que puede derivar en acciones específicas para la creación de una empresa. Modelos teóricos como el Evento Emprendedor (Shapero y Sokol, 1982) y la Teoría de la Conducta Planificada (Ajzen, 1991) han servido de marco de referencia (Krueger, 1993; Krueger y Brazeal, 1994; Krueger et al., 2000; Liñán y Chen, 2009).

Desde una consideración más amplia, Shane y Venkataraman (2000) sitúan el emprendimiento más allá del estudio de los factores personales asociados a la emergencia de nuevas empresas. Para estos autores supone el estudio de las fuentes, los procesos y las personas involucradas en el descubrimiento, la evaluación y la explotación de las oportunidades que se generan en bienes y servicios. El presente estudio adopta este enfoque al considerar que los factores contextuales al igual que los factores personales pueden ser decisivos. Podemos mencionar el estudio realizado por Lüthje y Franke (2003) en el que se reveló que los factores contextuales relacionados con los obstáculos y los apoyos percibidos para crear una empresa afectaban a la preferencia por seguir una trayectoria empresarial, al mismo tiempo que moderaban la relación actitud-intención. Así, en aquellos casos en que se percibía un contexto hostil, los estudiantes no mostraban su deseo de iniciar una empresa pese a su actitud favorable hacia el autoempleo. Para estos autores las barreras y las acciones de apoyo percibidas tienen un importante papel en la conducta empresarial de los estudiantes. Con lo cual se puede afirmar que la creación de una empresa ocurre en un contexto socioeconómico y cultural donde son relevantes la identificación de las oportunidades y la viabilidad de un proyecto empresarial frente a la consideración de los riesgos potenciales.

En cualquier época de crisis económica se producen cambios profundos e inesperados en la vida de las personas. En este contexto, las acciones concernientes a la adquisición de un trabajo y la forma de cómo conseguir una remuneración que garantice mantener un estatus social están rodeadas por la incertidumbre que se incrementa por la masiva des- trucción de empleo. Dichos cambios generan malestar en las personas cuando perciben que no pueden afrontar de forma adecuada estas situaciones (Lazarus y Folkman, 1984). Varios estudios indican que el desempleo afecta a las personas en su salud y bienestar psicológico provocando estrés, ansiedad e ira, a su vez, estas personas son más vulnerables a otros eventos vitales estresantes. Las circunstancias que rodean al desempleo, como un contexto socioeconómico desfavorable con recesión económica, distribución desigual de los recursos o falta de protección social, contribuyen a intensificar los efectos negativos (Kessler, House y Turner, 1987; McKeeRyan, Song, Wanber y Kinicki, 2005; Paul y Moser, 2009; Ünal-Karagüven, 2009). En términos concretos, el desempleo a nivel micro es un indicador de estrés, a nivel macro es una característica de crisis económica, depresión y recesión (Brenner y Mooney, 1983). Frente al impacto negativo que provoca la crisis económica actual, cabe también la posibilidad de que se desencadene una apreciación de eustres (Selye, 1984) si la persona es capaz de valorar una serie de beneficios u oportunidades para el desarrollo a raíz de las experiencias vividas. En este último caso se podrían ubicar las personas emprendedoras que se esfuerzan por identificar y aprovechar las oportunidades del entorno para salir de la inestabilidad económica. Por esta razón, consideramos oportuno incorporar una línea de trabajo que permita situar el emprendimiento en el contexto de crisis económica actual y poder determinar el impacto que tiene este evento sobre el emprendimiento. Para ello puede ser pertinente la Teoría de la Conservación de Recursos o Teoría COR formulada por Hobfoll (1998; 2001).

\section{Teoría de la Conservación de Recursos}

Hobfoll (1998; 2001) proporciona una teoría que asume los procesos internos (self) en la valoración de los eventos estresantes y los sitúa en un contexto social y cultural. Un supuesto básico de la teoría es que la gente se esfuerza en proteger, conservar y obtener más recursos. Recursos que son valorados por las personas en la medida en que contribuyen al mismo tiempo a la obtención de nuevos recursos, la promoción o protección de los que ya dispone. La teoría define el estrés como un proceso mediante el cual se produce una pérdida de los recursos o amenaza de su pérdida. Si las personas consiguen evitar y protegerse de la merma de recursos o bien recuperarse de este quebranto generando más recursos de los que ya se disponen, disminuirán los niveles de estrés percibido. De este modo, la pérdida genera una tensión favorable hacia la protección de los recursos pero no hacia la creación de nuevos recursos incrementando la vulnerabilidad a nuevas pérdidas. Por el contrario, las personas que obtienen más recursos son más proclives a generar nuevos recursos favoreciendo así una espiral de ganancias.

Según la Teoría COR la pérdida de recursos es el factor clave del estrés percibido, siendo más potente y decisivo que la ganancia de recursos, según dos principios generales: el principio de prominencia de la pérdida de recursos frente a 
la ganancia y el principio de inversión de recursos como protección o recuperación de las pérdidas y la obtención de nuevos recursos (Hobfoll, 2001). El principio de prominencia establece que, cuando la relación pérdida - ganancia es equivalente, la pérdida tendrá un mayor impacto, puesto que hay más agotamiento de recursos después de una pérdida que generación de recursos ante la ganancia. También señala que la trascendencia de la ganancia se concibe en el contexto de pérdida de recursos. Varios estudios permiten confirmar estas afirmaciones (Ennis, Hobfoll y Schröeder, 2000; Wells, Hobfoll y Lavin, 1999). Por su parte, el citado principio de inversión de recursos, pone de manifiesto el potencial impacto positivo de los eventos estresantes, ya que el motor de éste es la protección y recuperación de la pérdida de recursos, de tal manera que la pérdida, por ejemplo, de recursos económicos no sólo actúa como un evento estresante, sino también como un factor crítico en la puesta en marcha de estrategias de afrontamiento que implican la obtención de nuevos recursos, como ha puesto de manifiesto el estudio de ÜnalKaragüven (2009).

No podemos dudar que la crisis económica es un evento relacionado con la pérdida de los recursos relacionados con el empleo, lo que supone al mismo tiempo una reducción en las posibilidades de obtención de nuevos recursos. El trabajo implica una remuneración, un recurso muy importante para la seguridad y la estabilidad de la persona, pero además proporciona el acceso a otros recursos como el estatus social, la confianza en sí mismo, la autoestima, la oportunidad para el desarrollo de habilidades, el liderazgo, las redes sociales y el acceso a los recursos comunitarios (Brenner y Mooney, 1983). Desde esta perspectiva, la crisis económica como evento amenazante puede condicionar las acciones que realicen las personas para protegerse de las pérdidas o, por el contrario, optimizar y generar más recursos para afrontar la situación, de manera que alcancen a ver oportunidades para iniciar una actividad emprendedora, incluso en la adversidad. Para avanzar en esta línea de trabajo consideramos relevante examinar dos recursos personales que, en el marco de la Teoría COR, ofrecen la posibilidad de generar nuevos recursos que son decisivos para el emprendimiento en el contexto de crisis actual. El primero de ellos es la autoeficacia porque favorece la iniciativa y la identificación de oportunidades, el segundo es la creatividad porque sirve a la innovación y al aprovechamiento de las oportunidades.

\section{Autoeficacia y creatividad: Recursos personales para fomentar la identificación y aprovechamiento de re- cursos}

La autoeficacia se define como las "creencias en las propias capacidades para organizar y ejecutar los cursos de acción requeridos para obtener determinados logros" (Bandura, 1997, pág. 3). En esencia hace referencia a la confianza en la competencia personal para realizar una acción. Siguiendo a Bandura, las creencias de autoeficacia juegan un rol esencial en los procesos de autorregulación del nivel de motivación, los estados afectivos, los pensamientos y las acciones de las personas, al mismo tiempo que puede implicar la capacidad para manejar las circunstancias cambiantes de la vida. Las personas que se consideran eficaces tenderán a mostrar sentimientos optimistas sobre sus capacidades, podrán tomar decisiones sobre el curso de sus vidas y adoptarán sus objetivos personales. Además, actúa como amortiguador ante los eventos que generan estrés, si se infiere que las personas con elevados niveles de eficacia percibida tienden a interpretar las demandas y los problemas, como retos y no como amenazas. Conviene resaltar que las personas que creen en sus capacidades afrontan los desafíos con perseverancia, incrementando su nivel de esfuerzo y teniendo la absoluta seguridad de que los pueden controlar (Bandura, 1997, 1995/1999). Con todos estos argumentos, no hay duda que la autoeficacia es un recurso que reduce la vulnerabilidad al estrés (LeónRubio, Cantero y León-Pérez, 2011; Sánchez, Guillén y León-Rubio, 2006) y fortalece la resilencia ante situaciones adversas (Bandura, 2001), por consiguiente, la inclusión de la autoeficacia se cataloga como decisiva en esta investigación.

Diferentes trabajos identifican el poder predictivo de la autoeficacia sobre la elección de la carrera empresarial, la intención y las acciones dirigidas a la creación de una empresa (Boyd y Vozikis, 1994; Chen, Green y Crick, 1998; Jung, Ehrlich, De Noble y Baik, 2001; Krueger y Brazeal, 1994; Krueger et al., 2000; Moriano, Palací y Morales, 2006a; Sánchez, 2009). En estos trabajos, el constructo de autoeficacia emprendedora se refiere a la creencia de la persona en que posee las capacidades y habilidades para desempeñar de manera eficaz los roles y las acciones específicas implicadas en la creación y el ejercicio de un actividad empresarial, proporcionando suficiente evidencia como para que Krueger señalara que ésta es el antecedente sustancial de la percepción de oportunidades: "si nos vemos como competentes tendremos más probabilidad de ver un curso de acción como factible, así veremos con mayor probabilidad una oportunidad" (Krueger, 2000, p. 7). Chen et al. (1998) situaron la autoeficacia emprendedora como una característica distintiva de la persona emprendedora, distinguiendo dos componentes de la misma que diferenciaban a los ejecutivos fundadores de su empresa del resto: innovación y asunción de riesgos. De ahí que nos hayamos interesado, de manera particular, por la autoeficacia y la creatividad; la confianza en las capacidades propias es imprescindible para asumir riesgos (vid. Stewart y Roth, 2001), y la capacidad para idear algo nuevo y original, al tiempo que útil y beneficioso, que suele considerarse la base de la innovación (vid. Boix y Lazzeretti, 2012).

$\mathrm{Al}$ igual que se plantea con la autoeficacia, podemos entender la creatividad como un recurso personal que interviene en los procesos de estrés si bien el tipo de relación es menos clara puesto que los resultados de las investigaciones han sido hasta ahora contradictorios. En unos casos se ha observado que los eventos adversos pueden causar un decremento del rendimiento creativo al demandar recursos cognitivos que no están disponibles en las personas, por el contrario, otros estudios concluyen que las situaciones de estrés pueden 
originar soluciones creativas al favorecer la estimulación cognitiva y la activación motivacional. Byron, Khazanchi y Nazarian (2010), en su meta-análisis de estudios experimentales, llegaron a plantear una tercera línea de trabajo al introducir nuevos elementos de análisis, señalando que para determinar los efectos que puede generar el estrés sobre la creatividad no sólo es importante considerar el nivel de demanda que genera una situación estresante sino también el tipo de demanda que plantea. Así, observaron que, en términos generales, las situaciones de bajo estrés incrementaba el desempeño creativo mientras que disminuía en situaciones de alto estrés. Al considerar el contexto evaluativo detectaron una relación curvilínea en forma $U$ invertida, donde la amenaza a la evaluación social incrementaba el desempeño creativo en comparación con las condiciones de control pero cuando esta amenaza era mayor disminuía el rendimiento. En los casos en que se consideraba la controlabilidad de la situación, se detectó que en aquellos contextos caracterizados por su incontrolabilidad disminuía el rendimiento creativo.

Profundizando más en el concepto, la creatividad se refiere a la generación de ideas originales, útiles y apropiadas. De conformidad con esta línea de pensamiento y con una mayor orientación hacia el desempeño creativo, Amabile (1983) sugiere que para que la respuesta a un problema se considere creativa debe ser novedosa, apropiada, útil, correcta y valiosa. Además, se requiere que sea heurística en el sentido de que no siga una ruta identificable de solución. En este sentido, la creatividad presupone una serie de procesos cognitivos en los que subyacen dominios específicos como procesamiento relacional, independencia de campo o control cognitivo en el proceso atencional (Hennessey y Amabile, 2010). Situándonos en el contexto empresarial, la creatividad es considerada la capacidad de partida para el proceso de innovación (Zhou y George, 2001), entendido éste como un conjunto de etapas sucesivas necesarias para crear nuevos productos y métodos de producción, abrir nuevos mercados y desarrollar nuevas fuentes de suministro de materias primas y otros insumos, y nuevas formas de organización (León-Rubio, 2013). Con estos argumentos podemos sugerir que la creatividad en el emprendimiento es fundamental, teniendo en cuenta que para el desarrollo de iniciativas emprendedoras son necesarios el aprovechamiento de las oportunidades y la resolución de tareas en donde las personas deben encontrar la mejor manera de llevar a buen término su proyecto. Aquí, se puede tomar como referencia el estudio de Zampetakis y Moustakis (2006), donde se encontró evidencia de que la auto-percepción creativa de los estudiantes de ingeniería ejercía un efecto positivo sobre la intención emprendedora. También es importante considerar los estudios que vienen a señalar cómo ciertas características del entorno organizacional, como la presión del tiempo, la presencia de acciones de apoyo a la creatividad o la autonomía, pueden condicionar el proceso creativo en las personas (Hennessey y Amabile, 2010), que va en línea de lo que señalan Byron et al. (2010). Todo lo cual nos lleva a plantear en qué medida la presencia de eventos que generan pérdida de recursos pueden activar mecanismos cognitivos y motivacionales que promuevan la creatividad e impulsen la intención emprendedora.

\section{Planteamiento del problema e hipótesis}

Conforme a la literatura revisada, este estudio plantea una línea de trabajo del emprendimiento desde una perspectiva psicosocial que incorpora variables situacionales y personales basándonos en los principios de la Teoría COR (Hobfoll, 1998; 2001). Por un lado, se pretende estudiar el efecto que ejercen factores de estrés relacionados con la pérdida, amenaza de pérdida o ganancia de recursos derivados de la crisis económica sobre la intención de crear una empresa o negocio en estudiantes universitarios de posgrado. Se puede argumentar que las personas que consideran más las pérdidas frente a las ganancias serán más cautas a la hora de plantearse la creación de una empresa como opción de salida a una situación de crisis económica (Ünal-Karagüven, 2009). Mientras que, las personas que poseen más recursos se encuentran en una mejor posición para generar más recursos (Ennis et al., 2000; Wells et al., 1999), con lo cual puede verse incrementada la motivación para tomar la iniciativa de crear una empresa. Por otro lado, se quiere analizar el papel moderador que pueden ejercer la autoeficacia emprendedora (Krueger, 2000) y la percepción de la propia creatividad (Zampetakis y Moustakis, 2006), en el sentido de que pueden alterar las relaciones establecidas al incrementar las posibilidades de que los estudiantes que posean estos recursos personales muestren mayor intención emprendedora. Como hipótesis se formularon las siguientes:

Hipótesis 1. La evaluación global de recursos estará relacionada con la intención emprendedora en estudiantes universitarios de posgrado de manera que:

Hipótesis 1.1. La evaluación de pérdida de recursos o amenaza de pérdida reducirá la intención emprendedora.

Hipótesis 1.2. La evaluación de ganancia de recursos incrementará la intención emprendedora.

Hipótesis 2. La relación entre la evaluación de pérdida, amenaza de pérdidas o ganancia de recursos e intención emprendedora estará moderada por la autoeficacia emprendedora y la percepción creativa de los estudiantes universitarios de posgrado, donde:

Hipótesis 2.1. La autoeficacia emprendedora debería minimizar los efectos de la pérdida y de la amenaza de pérdida de recursos sobre la intención emprendedora.

Hipótesis 2.2. La autoeficacia emprendedora debería incrementar los efectos de la ganancia de recursos sobre la intención emprendedora.

Hipótesis 2.3. La percepción de la propia creatividad debería minimizar los efectos de la pérdida y de la amenaza de pérdida de recursos sobre la intención emprendedora. 
Hipótesis 2.4. La percepción de la propia creatividad debería incrementar los efectos de la ganancia de recursos sobre la intención emprendedora.

\section{Método}

\section{Participantes}

En el estudio participaron 278 estudiantes de másteres oficiales y propios de diferentes disciplinas ofertadas por la Universidad de Sevilla (Psicología, Arquitectura, Ciencias del Trabajo, Economía y Turismo). Cabe resaltar la pertinencia de utilizar esta población puesto que los estudiantes universitarios suelen considerarse como potenciales emprendedores en comparación con otras poblaciones (Krueger et al., 2000; Liñán, Urbano y Guerrero, 2011; Lüthje y Franke, 2003). El estudio de campo se llevó a cabo en los meses de mayo y junio de 2010. El 39.6\% de los participantes fueron hombres y el $60.4 \%$ mujeres, en un rango de edad de los 20 a los 51 años $(M=26.57, S D=5.167)$. Un porcentaje elevado residían con la familia (58.6\%), el resto con su pareja (13.3\%), solos $(9.7 \%)$ o en otra situación diferente a la solicitada $(18.3 \%)$. La gran mayoría se situaron en el nivel socio económico medio (69.1\%) frente al $12.6 \%$ de nivel medio-alto y el $15.5 \%$ de nivel socioeconómico medio-bajo, en el nivel bajo se situó el 1,8\% frente al $0.7 \%$ de nivel alto. Con respecto a la situación laboral, el 34.5\% trabajaban en el momento del estudio y el $65.5 \%$ no trabajaban.

\section{Procedimiento}

Se accedió a la muestra tras una previa solicitud a la coordinación de los respectivos másteres para contar con su colaboración y requerirles un espacio de tiempo óptimo para poder aplicar de forma colectiva el cuestionario en sus correspondientes aulas y en horario de clases. Una vez allí se pidió al alumnado la colaboración voluntaria, comunicándoles que se trataba de un estudio que pretendía analizar diversas cuestiones relacionadas con la crisis económica y su futuro laboral. Se veló en todo momento por la confidencialidad de la información.

\section{Instrumentos}

Evaluación de la Conservación de Recursos. Se utilizó esta escala para determinar cómo afectaba la crisis económica en términos de pérdidas, amenazas de pérdidas o ganancias de recursos. En su formato original la componen 74 ítems o recursos clave (Hobfoll, 1998; 2001). En nuestro estudio se seleccionaron un total de 20 reactivos relacionados con la situación socioeconómica actual que incluyen tanto situaciones (por ejemplo, estabilidad financiera, empleo estable o dinero para mejorar mi situación) como condiciones personales (por ejemplo, habilidad para organizar las tareas o reconocimiento de mis responsabilidades). Para cada uno de estos reactivos los participantes valoraban en una es- cala de 0 (no aplicable) a 4 (en un alto grado) la medida en que éstos han supuesto en los últimos seis meses una pérdida real, esto es, cuando se ha reducido la disponibilidad del recurso, una amenaza de pérdida que se considera al tener indicios de posible pérdida de recurso pero que no se ha producido aún, o una ganancia del recurso. La subescala que medía la ganancia de recurso obtuvo el coeficiente consistencia interna de Cronbach más alto $(\alpha$ $=.85)$ frente a los que se obtuvieron en las subescalas de pérdida $(\alpha=.78)$ y amenaza de pérdida que fue el más bajo $(\alpha=.69)$, lo que nos obliga a interpretar con cautela los datos de esta subescala.

- Autoeficacia emprendedora. Se empleó la escala de autoeficacia del cuestionario de Valores e Intenciones Empresariales (Rueda, Moriano y Liñán, 2011). Esta escala se aplica para determinar el grado en la que persona se cree capaz de realizar ciertas tareas concernientes a la creación de una empresa o negocio. La escala utilizada en este estudio la conforman seis ítems que contemplan las etapas y cuestiones primordiales del proceso emprendedor, entre los que podemos mencionar: definir mi idea de negocio y la estrategia de una nueva empresa, reconocer oportunidades en el mercado para nuevos productos $y /$ o servicios, crear y poner en funcionamiento una nueva empresa. Los participantes valoraron cada uno de los ítems en una escala de medida de 0 a 6 (totalmente ineficaz o totalmente eficaz). El coeficiente de consistencia interna de Cronbach fue .89.

Percepción creativa. Se adaptó la escala desarrollada por Zhou y George (2001) que tiene por objetivo determinar el grado en que determinadas conductas creativas se presentan en la persona. La escala original la componen 13 ítems que indican diferentes características que los supervisores pueden identificar de la conducta de sus empleados. Se seleccionaron cuatro ítems que hacían referencia específica a dicho comportamiento en el ámbito organizacional y se referían a la producción de ideas creativas (Zampetakis, 2008), como: muestro mi creatividad cuando tengo la oportunidad de hacerlo, me considero una buena fuente de ideas creativas. Se valoró en qué medida la persona se identificaba con cada una de las afirmaciones que se planteaban en una escala de 1 (no es característico en mí) a 5 (muy característico). Se obtuvo un coeficiente de consistencia interna de Cronbach de .81 .

Intención emprendedora (Rueda, Moriano y Liñán, 2011). Se utilizó esta escala para determinar la intención de convertirse en una persona emprendedora. Incluye cinco ítems, entre ellos: es muy probable que llegue a crear una empresa algún dia, estoy dispuesto a esforzarme lo que sea necesario para ser empresario, tengo serias dudas sobre si alguna vez. llegaré a crear una empresa. Para cada uno de los ítems que componía la escala se valoraba de 0 (nada) a 6 (totalmente). El análisis de fiabilidad proporcionó un $\alpha=.88$.

Se incluyeron una serie de preguntas relativas a la edad, sexo, personas con las que reside (sólo, familia, pareja, otros), el grupo socioeconómico al que pertenece (bajo, 
medio-bajo, medio, medio-alto, alto) y situación laboral (trabaja, no trabaja).

\section{Análisis de datos}

En primer lugar se procedió a la depuración de la muestra identificándose los valores extremos. Se detectó un caso en la variable pérdida de recursos por lo que se excluyó en los análisis posteriores. Con la muestra resultante de 277 participantes se llevaron a cabo análisis descriptivos y se calculó el coeficiente Alpha de Cronbach de los distintos instrumentos de medida. Posteriormente se hallaron las correlaciones bivariadas entre las variables objeto de estudio mediante el coeficiente de correlación de Pearson.

Para la comprobación de las hipótesis 1.1 y 1.2, se realizó un primer análisis de regresión múltiple para determinar el valor predictivo de las tres variables contempladas en la escala de Evaluación de la Conservación de Recursos, consideradas al mismo tiempo, con la puntuación total de la variable intención emprendedora. Para disminuir el efecto de colinealidad, todas las variables introducidas en el modelo se centraron a sus medias. En el análisis se incluyeron como variables de control el sexo (variable dummy con valor 0 a Hombre y 1 a Mujer) y la edad.

Después de realizar el primer análisis de regresión múltiple y con objeto de comprobar las hipótesis 2.1, 2.2, 2.3 y 2.4, se decidió llevar a cabo un segundo análisis de regresión múltiple siguiendo el método de pasos sucesivos ajustándose al principio de parsimonia en la predicción de la variable dependiente. En el análisis se introduce la puntuación total de la escala intención emprendedora como variable dependiente y, como explicativas, las variables pérdida, amenaza de pérdida, ganancia de recursos, autoeficacia emprendedora y percepción creativa. Al mismo tiempo y con el objeto de evaluar el efecto de interacción, se introdujo en el modelo la interacción entre las tres variables de la escala de Evaluación General de Recursos con las variables autoeficacia emprendedora y percepción creativa, se crean de este modo seis nuevas variables producto de las variables anteriormente mencionadas. Como criterio de entrada y salida se consideró que: (a) una variable es incorporada al modelo si su coeficiente de regresión parcial es significativamente distinto de cero con un nivel de significación del 5\%; y (b) una vez seleccionada, sólo es eliminada del modelo si con la incorporación de una u otras variables en un paso posterior, su coeficiente de regresión parcial deja de ser distinto de cero, con un nivel de significación del $10 \%$. Siguiendo el mismo procedimiento de antes, las variables introducidas fueron centradas para evitar problemas de multicolinealidad. Al igual que en el primer análisis de regresión también se controlaron los efectos del sexo y la edad. En todos los análisis se utilizó el programa estadístico SPSS versión 18.0.

\section{Resultados}

Los resultados descriptivos y correlaciones entre las variables examinadas se presentan en la Tabla 1. Como puede verse, la pérdida de recursos en la muestra de estudiantes es baja considerando la máxima puntuación total posible (80 puntos), siendo superada por la amenaza percibida de pérdida y la ganancia de recursos. Acorde a los valores promedio en autoeficacia emprendedora, percepción creativa e intención emprendedora, se obtienen puntuaciones moderadas en esta última variable, mientras que en las dos primeras son algo más elevadas. La matriz de correlación nos permitió averiguar qué parámetros presentaban un grado de asociación significativa con la intención emprendedora. De las variables examinadas, tres de ellas presentaban una correlación positiva significativa con la variable criterio intención emprendedora que fueron ganancia de recursos, autoeficacia emprendedora y percepción creativa. Se presentaron también correlaciones en ambos sentidos entre las tres variables contempladas en la escala de Evaluación de la Conservación de Recursos (pérdida, amenaza de pérdida y ganancia de recursos). Por último, entre las variables autoeficacia emprendedora y percepción creativa se encontró una correlación significativa positiva.

Tabla 1. Media, desviaciones estándar y correlaciones.

\begin{tabular}{|c|c|c|c|c|c|c|c|}
\hline & $M$ & $S D$ & 1 & 2 & 3 & 4 & 5 \\
\hline $\begin{array}{l}\text { 1. Pérdida de } \\
\text { recursos }\end{array}$ & 7.92 & 8.75 & - & & & & \\
\hline $\begin{array}{l}\text { 2. Amenaza de } \\
\text { pérdida }\end{array}$ & 12.49 & 8.26 & $.13^{*}$ & - & & & \\
\hline $\begin{array}{l}\text { 3. Ganancia de } \\
\text { recursos }\end{array}$ & 25.81 & 13.98 & $-.47 * *$ & $-.48 * *$ & - & & \\
\hline $\begin{array}{l}\text { 4. Autoeficacia } \\
\text { emprendedora }\end{array}$ & 19.97 & 6.70 & -.08 & .01 & $.25^{* *}$ & & \\
\hline $\begin{array}{l}\text { 5. Percepción } \\
\text { creativa }\end{array}$ & 13.86 & 3.03 & -.00 & -.06 & $.26 * *$ & $.37 * *$ & - \\
\hline $\begin{array}{l}\text { 6. Intención } \\
\text { emprendedora }\end{array}$ & 14.90 & 7.16 & .01 & -.07 & $.29 * *$ & $.42 * *$ & $.29 * *$ \\
\hline
\end{tabular}

En lo que respecta al primer análisis de regresión efectuado, la incorporación de las variables pérdida, amenaza y ganancia de recursos junto con las variables de control explican un $16.9 \%\left(R^{2}=.16\right)$ de la varianza de la variable intención emprendedora $(F=11.01, p<.001)$. Como se muestra en la Tabla 2, las variables pérdida y ganancia de recursos contribuyen de forma significativa al ajuste del modelo. A pesar de que el coeficiente para la variable amenaza no obtuvo un resultado significativo $(\phi=.06)$, se mantuvo en el modelo. Observando los coeficientes Beta, puede comprobarse que la variable que más peso tiene en la ecuación es la ganancia de recursos, después la pérdida de recursos y en tercer lugar el sexo. El resultado positivo en la relación señala que a medida que se incrementan las ganancias o las pérdidas en recursos, mayor es la intención empresarial. Ocurre lo contrario con la variable sexo indicando que la intención emprendedora es menor en el caso de las mujeres. Una vez calculados los es- 
tadísticos de colinealidad, se observó que el mayor índice de condición fue igual a 2.88 lo que indica la no existencia de problemas de multicolinealidad que altere la estimación de los resultados obtenidos. En vista de los resultados de este primer modelo podemos decir que sólo se cumple la Hipótesis 1.2 .

Tabla 2. Regresión múltiple para la variable intención emprendedora.

\begin{tabular}{lllll}
\hline Variable & $B$ & SE $B$ & $\beta$ & $t$ \\
\hline Sexo & -3.00 & .81 & -.20 & $-3.68^{* * *}$ \\
Edad & 0.04 & .07 & .03 & 0.56 \\
Pérdida & 0.18 & .05 & .22 & $3.47^{* *}$ \\
Amenaza & 0.10 & .05 & .12 & 1.92 \\
Ganancia & 0.22 & .03 & .43 & $5.93^{* * *}$ \\
\hline Nota: $* * p<.01 ; * * \neq<.001$ & & &
\end{tabular}

En el segundo análisis de regresión siguiendo el método de pasos sucesivos, fueron necesarios cuatro pasos para la configuración del modelo de regresión. En el primer paso se incorporó sólo la variable autoeficacia emprendedora con una proporción de $.18\left(\mathrm{R}^{2}\right)$ de la varianza de la puntuación en intención empresarial $(F=61,90, p<.001)$. En el segundo paso, se incorporó la interacción ganancia y percepción creativa, con un incremento en $R^{2}$ de .23 que contribuyó a mejorar el ajuste de forma significativa $(F=16.63, p<.001)$. En el tercer paso, se incorporó la interacción pérdida de recursos y percepción creativa cuyo valor $\mathrm{R}^{2}$ aumentó hasta $.25(\mathrm{~F}=$ $8.53, p<.05)$. En el cuarto y último paso, se incorporó la variable sexo $(F=8.74, p<.05)$. Las cuatro variables seleccionadas en el modelo final consiguen explicar un $27.7 \%\left(R^{2}=.27\right)$ el comportamiento de la variable intención empresarial. Como puede verse en la Tabla 3, la autoeficacia emprendedora

Tabla 3. Regresión múltiple (método pasos sucesivos) para la variable intención emprendedora.

\begin{tabular}{lllll}
\hline Variable & $B$ & SE B & $\beta$ & $t$ \\
\hline Paso 1 & & & & \\
$\quad$ Autoeficacia emprendedora & 0.45 .05 & .42 & $7.86^{* * *}$ \\
Paso 2 & & & & \\
$\quad$ Autoeficacia emprendedora & 0.37 .06 & .35 & $6.24^{* * *}$ \\
$\quad$ Ganancia x Percepción creativa & 0.00 .00 & .23 & $4.07^{* * *}$ \\
Paso 3 & & & \\
$\quad$ Autoeficacia emprendedora & 0.35 .06 & .33 & $6.01^{* * *}$ \\
$\quad$ Ganancia x Percepción creativa & 0.00 .00 & .28 & $4.85^{* * *}$ \\
$\quad$ Pérdida x Percepción creativa & 0.00 .00 & .16 & $2.92^{* *}$ \\
Paso 4 & & & \\
$\quad$ Autoeficacia emprendedora & 0.34 .05 & .31 & $5.75^{* * *}$ \\
$\quad$ Ganancia x Percepción creativa & 0.00 .00 & .27 & $4.74^{* * *}$ \\
$\quad$ Pérdida x Percepción creativa & 0.00 .00 & .16 & $3.01^{* *}$ \\
Sexo & -2.25 .76 & -.15 & $-2.95^{* *}$ \\
\hline ** $p<.01 ; * * *<.001$ & & &
\end{tabular}

es la variable que tiene mayor fuerza predictiva, mientras que los términos interactivos ganancia-percepción creativa y pérdida-percepción creativa junto con la variable sexo contribuyen a mejorar la capacidad explicativa del modelo. Acorde a los resultados de la regresión, la autoeficacia emprendedora tiene un efecto positivo sobre la intención empresarial. Los valores positivos de los coeficientes de la interacción, indican que el efecto positivo de la ganancia o la pérdida de recursos sobre la intención empresarial es mayor cuando se unen el efecto de la percepción creativa. Una vez más, la intención emprendedora es menor dentro del grupo de las mujeres.

Finalmente, se observó que el mayor índice de condición obtenido, una vez calculados los estadísticos de colinealidad, se situó en 2.86 por lo que se consiguió disminuir el efecto de la multicolinealidad. En vista de los resultados podemos decir que sólo se cumplen parcialmente las Hipótesis 2.3 y 2.4.

\section{Discusión}

Los resultados obtenidos respecto a los factores relacionados con el emprendimiento en un contexto de crisis económica son tentadores pese a que las hipótesis propuestas no se hayan podido confirmar con total claridad.

En primer lugar, sólo se pudo confirmar la Hipótesis 1.2 al cumplirse la predicción sobre la ganancia de recursos y la intención emprendedora. Acorde con la Teoría COR (Hobfoll, 1998; 2001) se puede concluir que las personas que poseen más recursos son más capaces de generar nuevos recursos por lo que es más probable que sea entre estas personas quienes se planteen iniciar su trayectoria como emprendedoras. El hecho de que la variable pérdida de recursos contribuyera de forma significativa a explicar la intención emprendedora en la dirección opuesta a la planteada en la hipótesis de estudio requiere una mayor atención. En el presente estudio, los participantes puntuaron más en ganancia de recursos que en amenaza de pérdida de recursos que, a su vez, fue superior a la pérdida real que presentó una puntuación baja en general. Considerando los resultados obtenidos en otros estudios (Ennis et al., 2000; Wells et al., 1999) se puede entender que los efectos de la pérdida a corto plazo no presenten un efecto negativo sobre la intención emprendedora, si consideramos el momento en que se realizó el estudio y la población encuestada. El estudio se realizó en los primeros años de la crisis económica que, si bien tuvo un impacto global, afectó de manera particular a sectores económicos muy concretos como fue la construcción y otros sectores asociados a éste. No obstante, al contemplar la asociación positiva entre las variables pérdida y amenaza de pérdida de recursos y la asociación negativa entre ambas variables con ganancia de recursos, se puede anticipar que cuando se entra en un periodo prolongado de crisis económica es probable que éste pueda causar un coste mayor de recursos. De tal forma que, la persona que comienza a experimentar pérdidas es más vulnerable a las pérdidas futuras encontrándose en una espiral continua de pérdidas que afectaría a los mecanismos de afrontamiento a menos que se disponga de los recursos con que romper el ciclo negativo.

Otra línea argumental que podemos incluir aquí es su posible relación con los factores motivacionales que pueden estar involucrados en la creación de una empresa, donde por lo general se distinguen entre factores "push" o factores que fuerzan al desarrollo de la actividad emprendedora por nece- 
sidad (por ejemplo, encontrarse en desempleo o la falta de oportunidades) y factores "pull" más centrados en la atracción al considerar el emprendimiento una oportunidad de crecimiento o posibilidad de alcanzar cierta autonomía en el trabajo. Con respecto a esta distinción entre factores motivacionales por necesidad frente a factores por estímulo y atracción, es preciso señalar que recientes trabajos apuntan la dificultad de poder separarlos e incluso es más que probable que se dé una combinación de ambos (Dawson, y Henley, 2012). Así, se explicaría que tanto en condiciones de pérdida como ganancias se observe la intención emprendedora. Considerando los resultados de nuestro estudio, cabe la posibilidad de plantear el argumento de que las condiciones socioeconómicas de disponibilidad de recursos pueden estar detrás de los factores motivacionales de manera que ante la percepción de pérdida de recursos las personas se ven en la necesidad de crear una empresa como una alternativa a una situación desfavorable actual mientras que la percepción de ganancia de recursos incentivaría la conducta emprendedora desde una perspectiva de crecimiento y desarrollo.

En segundo lugar, se confirma el papel clave de la autoeficacia emprendedora, siendo la variable más influyente y decisiva en el estudio de la intención emprendedora (Krueger et al., 2000; Liñán y Chen, 2009). Además se proporciona evidencia empírica de que la autoeficacia en circunstancias potencialmente estresantes como la crisis económica constituye un elemento diferenciador en la percepción de oportunidades frente a las amenazas (Krueger, 2000). En el caso de la variable percepción creativa, sólo se encontró su influencia positiva cuando aparecía en interacción con la pérdida y con la ganancia de recursos. Si se entiende por creatividad la generación de soluciones novedosas, útiles y apropiadas puede explicarse su relación al mismo tiempo con estas variables, que puede ir en línea con lo señalado respecto a los mecanismos que intervienen en la inversión de los recursos (Hobfoll, 2001) y la intensidad del estresor (Byron et al., 2010). De este modo, se puede interpretar que en situaciones de pérdida real, la creatividad actuaría como mecanismo de protección y optimización de los recursos de la persona, dados los valores de moderados a bajos en pérdidas, mientras que en situaciones de ganancia la creatividad actuaría para la inversión y obtención de nuevos recursos. En este punto, hay que volver a mencionar el estudio de Chen et al. (1998) que contemplaron la innovación como factor que abarca un conjunto de roles y tareas relacionadas con la conducta de emprendimiento, entre estas tareas se encuentra la producción de nuevas ideas. De hecho en el presente estudio la autoeficacia emprendedora y la percepción creativa presentaron una correlación positiva significativa. En línea con las conclusiones del estudio realizado por Byron et al. (2010), se puede interpretar que la autoeficacia puede promover el efecto moderador beneficioso de la creatividad sobre la intención emprendedora ante la pérdida de recursos, al generar una evaluación de controlabilidad del evento, teniendo en cuenta que en el estudio esta pérdida no es elevada. Por esta razón, hay que considerar la relevancia que puede tener la creatividad en un contexto de crisis económica, donde la demanda de soluciones creativas para hacerle frente es indispensable. En definitiva, se entiende que la creatividad genera ideas novedosas y útiles tanto para la obtención de nuevos recursos como para un mejor aprovechamiento y optimización de los recursos existentes siempre que la persona se considere capaz.

Dentro de las limitaciones, hay que tener presente que este estudio se centra en la intencionalidad de la conducta. Thompson (2009) dejó claro que la intención emprendedora es un componente necesario entre quienes pretenden iniciarse en la carrera empresarial, pero esta intención no tiene por qué convertirse en una conducta real en el futuro. Según este planteamiento, si una de las personas encuestadas manifiesta su intención de crear una empresa en el momento del estudio, cabe la posibilidad de que finalmente opte por una vía completamente diferente a la carrera empresarial. De hecho, ha sido una circunstancia que ha estado presente en gran parte de los estudios que siguen una metodología transversal y sólo con estudios longitudinales es posible clarificar la tendencia en el emprendimiento de las personas que aún no han desarrollado su carrera como emprendedores. También es necesario apuntar la limitación que se deriva al realizar un estudio transversal basado únicamente en medidas de autoinforme para un único informante por lo que no cabe descartar el efecto de la varianza del método común. Una vez más se recomienda la realización de estudios donde se puedan combinar diferentes medidas al menos en dos fases. Otra limitación fue el hecho de que las puntuaciones obtenidas en la escala de evaluación de recursos no hubieran podido discriminar mejor los resultados obtenidos, observándose sólo la influencia positiva sobre la intención empresarial. Como ya se ha explicado, puede que la percepción de pérdida no sea experimentada con la intensidad suficiente como para que puedan preverse los efectos señalados en la primera hipótesis.

Finalmente los resultados parecen respaldar las diferencias detectadas en varios estudios en lo que se refiere al emprendimiento entre hombres y mujeres, las mujeres muestran menos intención emprendedora en comparación con los hombres (Schoon y Duckword, 2012; Sullivan y Meek, 2012). Con este dato, no cabe descartar la posibilidad de que el efecto de las variables consideradas en este estudio sobre la intención emprendedora sea diferente en hombres y mujeres. Por esta razón, se plantea la necesidad de analizar de forma más precisa las variaciones del género con respecto a la conducta emprendedora en función de la disponibilidad de los recursos. También se considera necesario detectar las razones que llevan a las mujeres a comprometerse menos en la actividad empresarial, al mismo tiempo que convendría investigar en qué momento y bajo qué circunstancias sería posible observar comportamientos contrarios a lo esperado en el colectivo de mujeres, esto es, una disposición favorable hacia la actividad empresarial (Verheul, Thurik, Griloy y van der Zwan, 2012). 
Entendemos que la circunstancia actual de crisis económica que se está prolongando y que está provocando la destrucción masiva de empleo, puede generar un ciclo de pérdidas (como el estatus económico, los bienes de consumo, optimismo y confianza), que afectará sobremanera al bienestar de las personas. Se plantea que las personas con falta de recursos adoptarán una postura defensiva y más conservadora de los recursos, mientras que la percepción de ganancia conllevará una estrategia más activa generadora de recursos que les puede ayudar a evitar las pérdidas y mantenerse en un ciclo de obtención de ganancias, donde la autoeficacia puede ser un elemento clave en este proceso. Acorde con los resultados obtenidos, las personas autoeficaces serán menos vulnerables a la pérdida de recursos y más capaces para orquestar la ganancia de recursos. Se podría continuar el trabajo analizando la dirección que tome la intención empresarial teniendo en cuenta, además de la disponibilidad de recursos, las estrategias de afrontamiento en contextos económicos desfavorables.

Hobfoll $(1998 ; 2001)$ considera que las personas organizan sus recursos personales y sociales desde su relación con los demás con el fin de hacer frente o ajustarse a una vida en circunstancias desfavorables. Razón por la cual, sitúa las estrategias de afrontamiento en el marco de una ecología social del contexto. Por ello consideramos adecuado para la continuación del estudio tomar en consideración el Modelo Multiaxial de Afrontamiento (Dunahoo, Hobfoll, Monnier, Hulsizer y Johnson, 1998; Hobfoll, 1998). De este modo se podría abordar la conducta de emprendimiento considerando las distintas maneras que tienen las personas de afrontar las circunstancias que se presenten en su trayectoria profesional, entre los que caben comportamientos prosociales o antisociales según se promueva la interacción social positiva o conlleve la rivalidad con otros que se perciben como obstáculos.

En la base del carácter social del afrontamiento se encuentra la distinción de los valores individualistas y colectivistas. De hecho, la influencia de los valores socialmente dominantes en una cultura se ha puesto en evidencia en estudios sobre la intención emprendedora (Jung et al., 2001; Moriano, Palací y Morales, 2006b). Se ha visto que la persecución de valores individualistas como poder, logro, hedonismo, estimulación y autodirección es una característica importante en el perfil psicosocial del emprendedor universitario (Moriano et al., 2006b). La limitación que pueden plantear estos estudios es el tipo de conducta emprendedora que han considerado hasta el momento, más competitiva y dirigida a la búsqueda de un beneficio propio. Por ello sería interesante también contemplar y diferenciar en los estudios de emprendimiento, los tipos de prácticas emprendedoras a las que una persona estaría dispuesta a optar. Si se plantean ciertos elementos diferenciadores entre el emprendimiento comercial, más orientado a una idea de negocio, frente al emprendimiento social, orientado a la creación de valor social frente al personal (Austin, Stevenson, Wei-Skillern, 2006; Renko, 2012), también se pueden inferir estrategias de afrontamiento diferentes teniendo en cuenta su carácter social.
Por otro lado, se ha señalado que las personas situadas dentro de una red de relaciones e intercambios se encuentran en una posición ventajosa para identificar oportunidades y obtener los recursos que son necesarios (Low y MacMillan, 1988). Por ello, puede ser también relevante incorporar la estructura social en el estudio del emprendimiento. Hablar de estructuras sociales, es hablar específicamente del capital social. En los estudios sobre emprendimiento, el capital social se valora considerando la posición social de las organizaciones, los vínculos de intercambio, las redes de contactos y las relaciones con los demás en términos de cooperación y confianza. El capital social proporciona redes que facilitan los flujos de conocimiento, el descubrimiento de oportunidades, una mayor cooperación y, por lo tanto, el acceso a los recursos que contribuyen al éxito (Davidsson y Honig, 2003; Markman y Baron, 2003). Es precisamente en esas redes y vínculos sociales, donde el individuo encuentra los recursos que le permiten vislumbrar oportunidades con miras a enfocar sus esfuerzos para la puesta en marcha de un proyecto de emprendimiento. Por ello se puede determinar hasta qué punto el capital social en el contexto socioeconómico actual puede ser relevante en la gestación de una actividad empresarial, ya sea económica o social.

De acuerdo con todo lo anterior, el camino a seguir en futuras investigaciones es afianzar la relación entre el estrés y el emprendimiento, puesto son pocos o inexistentes los estudios que la abordan y donde se ha visto que la teoría COR constituye un soporte teórico pertinente para esclarecer el emprendimiento en un contexto de crisis económica por el que atravesamos. Es conveniente que en futuras investigaciones se pueda clarificar y consolidar tal relación con análisis más específicos, incorporando la estructura social presente y las estrategias de afrontamiento, teniendo en cuenta los tipos de conducta de emprendimiento y adoptando una perspectiva de género. En estos estudios el abordaje longitudinal prospectivo aportaría la visión del emprendimiento como proceso en el tiempo.

A nuestro entender, este estudio es pionero al situarlo en el contexto del estrés, puesto que se analizan las condiciones bajo las cuales las personas ante la crisis económica pueden tomar la iniciativa de emprender una carrera empresarial propia. Entendemos que nuestra principal contribución es considerar el impacto que tiene un evento estresante como la situación económica que vivimos en el emprendimiento donde la autoeficacia y la creatividad son algo más que destrezas que se poseen. Se abre una nueva línea de trabajo en el estudio del estrés junto con las líneas ya consolidadas en el bienestar psicológico, la satisfacción y el rendimiento en el trabajo. Este trabajo no sólo puede ser interesante desde el punto de vista teórico, también plantea en cierta forma un reto para la promoción del emprendimiento, en las que caben medidas que abarcan desde la formación del emprendedor en el contexto educativo hasta el desarrollo de políticas activas para la generación de empleo en el contexto sociopolítico. Dado que el emprendimiento se está convirtiendo en una actividad vital para el desarrollo económico, se podría 
pensar en una educación más efectiva, donde se proporcione la formación de las competencias necesarias que contribuyan a fomentar el espíritu emprendedor considerando aquellos elementos que juegan un rol crítico en la decisión personal para iniciar una acción emprendedora. Rentabilizar la enseñanza de modo que genere futuros emprendedores sólo será posible si viene acompañada de políticas de impulso a la ac-

\section{Referencias}

Ajzen, I. (1991). The theory of planned behavior. Organizational Behaviour \& Human Decision Processes, 50, 179-211.

Amabile, T. M. (1983). The social psychology of creativity. New York: SpringerVerlag.

Austin, J., Stevenson, H. y Wei-Skillern, J. (2006). Social and commercial entrepreneurship: same, different, or both? Enterpreneurship Theory \& Practice, 30(1), 1-22.

Bandura, A. (1997). Self-efficacy: the exercise of control. New York: W.H. Free$\operatorname{man}$

Bandura, A. (1999). Ejercicio de la eficacia personal y colectiva en sociedades cambiantes. En A. Bandura (Ed.). Auto-eficacia: cómo afrontamos los cambios de la sociedad actual, (19-54). Bilbao: Desclée de Brouwer. (Traducido de Self-efficacy in changing societies, Ed. 1995. Cambridge University Press).

Bandura, A. (2001). Social cognitive theory: An agentic perspective. Annual Review of Psychology, 52, 1-26.

Baron, R. A. (2004). The cognitive perspective: A valuable tool for answering entrepreneurship's basic "why" questions. Journal of Business Venturing, 19, 221-239.

Boix, R. y Lazzeretti, L. (2012). Las industrias creativas en España: una panorámica. Investigaciones regionales, 22, 181-206.

Boyd, N. G. y Vozikis, G.S. (1994). The influence of self-efficacy on the development of entrepreneurial intentions and actions. Entrepreneurship Theory \& Practice, 18, 63-90.

Brenner, M. H. y Mooney, A. (1983). Unemployment and health in the context of economic change. Social Science \& Medicine, 17(16), 1138-1983.

Byron, K., Khazanchi, S. y Nazarian, D. (2010). The relationship between stressors and creativity: a meta-analysis examining competing theoretical models. Journal of Applied Psychology, 95(1), 201-212.

Chen, C. C., Greene, P. G. y Crick, A. (1998). Does entrepreneurial selfefficacy distinguish entrepreneurs from managers? Journal of Business Venturing, 13(4) 295-316.

Cromie, S. (2000). Assessing entrepreneurial inclinations: some approaches and empirical evidence. European Journal of Work \& Organizational Psychology, 9(1), 7-30.

Davidsson, P. y Honig, B. (2003). The role of social and human capital among nascent entrepreneurs. Journal of Business Venturing, 18, 301-331.

Dawson, C. y Henley, A. (2012). "Push" versus "pull" entrepreneurship: an ambiguous distinction? International Journal of Entrepreneurial Behaviour \& Research, 18(6), 697-719.

Dunahoo, C. L, Hobfoll, S. E., Monnier, J., Hulsizer M. R. y Johnson, R. (1998).There's more than rugged individualism in coping. Part 1: Even the lone ranger had tonto. Anxiety, Stress \& Coping: An International Journal, 11(2), 137-165.

Ennis, N. E., Hobfoll, S. E. y Schröeder, K. E. E. (2000). Money doesn't talk, it swears: How economic stress and resistance resources impact inner-city women's depressive mood. American Journal of Community Psychology, 28(2), 149-173.

Frank, H., Lueger, M. y Korunka, C. (2007). The significance of personality in business start-up intentions, start-up realization and business success. Entrepreneurship \& Regional Development: An International Journal, 19(3), 227-251

Gartner, W. B. (1988). "Who is an entrepreneur?" is the wrong question. American Journal of Small Business, 12(4), 11-32.

Hennessey, B. A. y Amabile, T. M. (2010). Creativity. Annual Review of Psychology. 61, 569-598. tividad emprendedora donde se contemplen dos objetivos estratégicos, que son, a nuestro modo de ver, generar recursos y oportunidades para que las iniciativas emprendedoras tengan garantías de éxito promoviendo la creencia de eficacia personal de quienes se inician en este sector de la economía y difundir experiencias exitosas e innovadoras de tal modo que favorezca la motivación personal y la identificación positiva.

Hobfoll, S. E. (1998). Stress, culture, and community: the psychology and philosophy of stress. New York: Plenum Press.

Hobfoll, S. E. (2001). The influence of culture, community, and the nested-self in the stress process: advancing conservation of resources theory. Applied Psychology: An International Review, 50(3), 337-421.

Jung, D. I., Ehrlich, S. B., De Noble, A. F. y Baik, K. B. (2001). Entrepreneurial Self-Efficacy and its Relationship to Entrepreneurial Actions: A Comparative Study Between the US and Korea. Management International, 6(1), 41-53.

Kessler, R. C., House, J. S. y Turner, J. B. (1987). Unemployment and health in a community sample. Journal of Health \& Social Behavior, 28 , $51-59$.

Krueger, N. F. (1993). The impact of prior entrepreneurial exposure on perceptions of new venture feasibility and desirability. Entrepreneurship Theory \& Practice, 18(1), 5-21.

Krueger, N. F. (2000). The cognitive infrastructure of opportunity emergence. Entrepreneurship Theory \& Practice, 24(3) 5-23.

Krueger, N. F. y Brazeal, D. V. (1994). Entrepreneurial potential and potential entrepreneurs. Entrepreneurship Theory \& Practice, 18(3), 91104

Krueger, N. F., Reilly, M. D. y Carsrud, A. L. (2000). Competing models of entrepeneurial intentions. Journal of Business Venturing, 15(5-6), 411-432.

Landström, H. (2008). Entrepreneurship research. A missing link in our understanding of the knowledge economy. Journal of Intellectual Capital, 9(2), 301-322.

Lazarus, R. S. y Folkman, S. (1984). Stress, appraisal \& coping. New York: Springer.

León-Rubio, J. M. (Febrero, 2013). Desenvolvimiento regional e empreendedorismo. Conferencia impartida en el XXXII Seminário G\&DR Internacional de Integração Econômica Global Brasil-Espanha. Taubaté, São Paulo, Brasil: Universidad de Taubaté.

León-Rubio, J. M., Cantero, F. J. y León-Pérez, J. M. (2011). Diferencias del rol desempeñado por la autoeficacia en el burnout percibido por el personal universitario en función de las condiciones de trabajo. Anales de Psicologia, 27(2), 518-526.

Liñán, F. y Chen, Y-W. (2009). Development and cross-cultural application of a specific instrument to measure entrepreneurial intentions. Enterpreneurship Theory \& Practice, 33(3), 593-617.

Liñán, F., Urbano, D. y Guerrero, M. (2011). Regional variations in entrepreneurial cognitions: Start-up intentions of university students in Spain. Entrepreneurship \& Regional Development, 23(3-4), 187-215.

Low, M. B. y MacMillan, I. C. (1988). Entrepreneurship: Past research \& future challenges. Journal of Management, 14(2) 139-161.

Lüthje, C. y Franke, N. (2003). The 'making' of an entrepreneur. Testing a model of entrepreneurial intent among engineering students at MIT". R\&D Management, 33(2), 135-147.

Markman, G. D. y Baron, R. A. (2003). Person-entrepreneurship fit: why some people are more successful as entrepreneurs than others. Human Resource Management Review, 13, 281-301.

McKee-Ryan, F. M., Song, Z., Wanber C. R. y Kinicki, A. J. (2005). Psychological and physical well-being during unemployment: A meta-analytic study. Journal of Applied Psychology, 90(1), 53-76.

Mitchell, R. K., Busenitz, L. W., Bird, B., Gaglio, C.M., McMullen, J. S., Morse, E. A. \& Smith, J. B. (2007). The central question in enterpreneurial cognition research 2007. Entrepreneurship Theory \& Practice, 31(1), $1-27$ 
Mitchell, R. K., Busenitz, L., Lant, T., McDougall, P. P., Morse, E. A. y Smith, J. B. (2002). Toward a theory of entrepreneurial cognition: Rethinking the people side of entrepreneurship research. Entrepreneurship Theory \& Practice, 27(2), 93-104.

Moriano, J. A., Palací, F. J. y Morales, J. F. (2006a). Adaptación y validación en España de la escala de Autoeficacia Emprendedora. Revista de Psicología Social, 21(1), 51-64.

Moriano, J. A., Palací, F. J. y Morales, J. F. (2006b). El perfil psicosocial del emprendedor universitario. Revista de Psicología del Trabajo y de las Organizaciones, 22(1), 75-99.

Paul, K. I. y Moser, K. (2009). Unemployment impairs mental health: Metaanalyses. Journal of Vocational Behavior, 74, 264-282.

Renko, M. (2012). Early Challenges of Nascent Social Entrepreneurs. Entrepreneurship Theory and Practice. Publicación anticipada en línea. doi: 10.1111/j.1540-6520.2012.00522.x.

Rueda, S., Moriano, J. A. y Liñán, F. (2011). Validating a theory of planned behavior questionnaire to measure entrepreneurial intentions. En F. Liñán, A. Fayolle y P. Kyro (Eds.), 8th ESU Conference on Entrepreneurship. Sevilla: Edición Digital @ Tres.

Sánchez, J.C. (2009). Aprendizaje social e intenciones emprendedoras: un estudio comparativo entre México, España y Portugal. Revista Latinoamericana de Psicología, 41(1), 109-119.

Sánchez, S., Guillén, C. y León-Rubio, J. M. (2006). La autoeficacia percibida en el afrontamiento de riesgos laborales psicosociales como variable moduladora del burnout. Ansiedad y Estrés, 12(2-3), 495-504.

Schoon, I. y Duckworth, K. (2012). Who becomes an Entrepreneur?. Early Life Experiences as Predictors of Entrepreneurship. Developmental Psychology, 48(6), 1719-1726.

Selye, H. (1984). The stress of life. New York: McGraw-Hill.

Shane, S. y Venkataraman, S. (2000). The promise of entrepreneurship as a field of research. Academy of Management Review, 25(1), 217-226.

Shapero, A. y Sokol, L. (1982). The social dimensions of entrepreneurship. En C. A. Kent, D. L. Sexton y K. H. Vesper (Eds.), Encyclopedia of Entrepreneurship (pp. 72-90). Englewood Cliffs, N.J.: Prentice-Hall.
Shaver, K. G. y Scott, L. R. (1991). Person, process, choice: the psychology of new venture creation. Entrepreneurship Theory \& Practice, 16(2), 23-45.

Stewart, W. H. y Roth, P. L. (2001). Risk propensity differences between entrepreneurs and managers: A meta-analytic review. Journal of Applied Psychology, 86(1), 145-153.

Sullivan, D.M. y Meek, W. (2012). Gender and entrepreneurship: a review and process model. Journal of Managerial Psychology, 27(5), 428-458.

Thompson, E.R. (2009). Individual entrepreneurial intent: construct clarification and development of an internationally reliable metric. Entrepreneurship Theory \& Practice, 33(3), 669-694.

Ünal-Karagüven, M. H. (2009). Psychological impact of an economic crisis: A Conservation of Resources Approach. International Journal of Stress Management, 16(3), 177-194.

Verheul, I., Thurik, R., Grilo, I. y van der Zwan, P. (2012). Explaining preferences and actual involvement in self-employment: Gender and the entrepreneurial personality. Journal of Economic Psychology, 33, 325-341.

Wells, J. D., Hobfoll, S. E. y Lavin, J. (1999): When it rains, it pours: The greater impact of resource loss compared to gain on psychological distress. Personality \& Social Psychology Bulletin, 25(9), 1172-1182.

Zampetakis, L. A. (2008). The role of creativity \& proactivity on perceived entrepreneurial desirability. Thinking Skills \& Creativity, 3, 154-162.

Zampetakis, L. A. y Moustakis, V. (2006). Linking creativity with entrepreneurial intentions: a structural approach. International Entrepreneurship os Management Journal, 2(3), 413-428.

Zhao, H., Seibert, S. E. y Lumpkin, G.T. (2010). The relationship of personality to entrepreneurial intentions and performance: A meta-analytic review. Journal of Management, 36(2), 381-404.

Zhou, J. y George, J. M. (2001). When job dissatisfaction leads to creativity: encouraging the expression of voice. Academy of Management Journal, 44(4), 682-696.

(Articulo recibido: 30-9-2012; revisado: 26-4-2013; aceptado: 4-5-2013) 